\title{
PELVIC CHONDROSARCOMA - THERAPEUTIC OPTIONS (LITERATURE REVIEW)
}

doi: $10.2478 /$ rojost-2018-0078

R. Popescu ${ }^{12}$, Șt. Cuculici' ${ }^{13}$, Șt. Cristea ${ }^{13}$

"'Carol Davila" University of Medicine and Pharmacy, Bucharest, Romania

"Foişor" Hospital of Orthopaedics, Trauma and OsteoarticularTB, Bucharest, Romania 3"Sf. Pantelimon" Emergency Hospital, Bucharest, Romania

Chondrosarcoma is a mesenchymal malignant tumor (primary or secondary) which is characterized by a cartilaginous mass formed out of neoplastic cells. It is one of the most frequent malignant bone tumors (after multiple myeloma and osteosarcoma), affecting more males than females (Ratio $M: F=2: 1$ ). It appears with predilection in the pelvis and long bones - mainly the proximal portion of the humerus and femur. It is a unique primary bone tumor through its chemotherapy and radiotherapy resistance, the only effective treatment being the surgical one. As far as the pelvis is concerned, it is important to determine the location, the dimension, and the relation with the nearby structures of the tumor, in order to establish if it is possible to do a complete excision of the tumor and adapt the subsequent reconstruction of the pelvis. In the excisions of the pelvic chondrosarcoma, the choices are conservatory surgery (limb saving) and the radical surgery (conventional pelvic resection). The oncological resection is the primary goal, but the functional result and the patient's quality of life are also very important.

Keywords: chondrosarcoma, resection, reconstruction, pelvis 